PII: S0038-1098(97)10010-2

\title{
ANOMALIES IN THE CATHODOLUMINESCENCE OF THE ANTIFERROMAGNETIC OXIDES NiO AND COO
}

\author{
C. Díaz-Guerra and J. Piqueras \\ Departamento de Física de Materiales, Facultad de Físicas, Universidad Complutense, E-28040 Madrid, Spain
}

(Received 8 April 1997; accepted 11 August 1997 by P. Burlet)

\begin{abstract}
Cathodoluminescence (CL) of the antiferromagnetic oxides $\mathrm{NiO}$ and $\mathrm{CoO}$ has been investigated. Both compounds show luminescence bands in the visible and near infrared spectral ranges, tentatively attributed to intra-ionic transitions. Dependence of the visible CL intensity with temperature presents anomalies at about half of the Néel temperature, which are explained by the existence of magnetic ordering effects. The results are briefly compared with previous reports on the $\mathrm{CL}$ from $\mathrm{YBa}_{2} \mathrm{Cu}_{3} \mathrm{O}_{7-x}$. (C) 1997 Elsevier Science Ltd
\end{abstract}

Keywords: A. magnetically ordered materials, A. insulators, D. optical properties, E. luminescence.

The transition metal monoxides $\mathrm{NiO}$ and $\mathrm{CoO}$ are antiferromagnetic electrically insulating ionic compounds with the rocksalt structure, whose insulating nature has been extensively investigated for many years. These and related oxides have been regarded as prototypes of Mottinsulators, but a series of experimental and theoretical studies indicate that their behaviour should be explained by a charge transfer mechanism. In particular, NiO has been considered as a charge transfer insulator with a gap of about $4 \mathrm{eV}$ (e.g. [1-4]), while the behaviour of $\mathrm{CoO}$ [5] indicates the existence of a gap of about $2.5 \mathrm{eV}$.

A significative part of the experimental results providing information on the energy gap of $\mathrm{NiO}$ and $\mathrm{CoO}$ refers to their optical absorption (e.g. [6-10]), while their optical emission properties have been scarcely investigated. Previous authors reported catodoluminescence (CL) emission of $\mathrm{NiO}$ with main bands at $2.5 \mathrm{eV}$ [11], attributed to a magnetic exciton state, or at about $4 \mathrm{eV}$ [12] which would correspond to the charge transfer gap energy. To our knowledge, not similar results on $\mathrm{CoO}$ are available. The luminescence of other transition-metal oxide, $\mathrm{MnO}$, has been studied in detail in the past years [13-15]. From the temperature dependence of the emission intensity and peak energy it was concluded that the luminescence centers in $\mathrm{MnO}$ are closely related to the magnetic ordering of the manganese spins. In particular, the result on this antiferromagnetic insulator were explained by the existence of a magnon assisted photoluminescence (PL) band below the Néel temperature $\left(T_{N}\right)$ and a short-wavelength magnon assisted band above $T_{N}$. Similar results were reported for other manganese compounds $[16,17]$.

In the present work $\mathrm{CL}$ from $\mathrm{NiO}$ and $\mathrm{CoO}$ is investigated and the results compared with the mentioned results relative to $\mathrm{MnO}$. In particular, the possibility that some features of the luminescence emission can be interpreted by magnetic ordering processes below $T_{N}$ has been considered. The influence of antiferromagnetic ordering on the optical absorption $[18,19]$ and reflectivity spectra [20] of NiO has been previously reported. Part of the present interest in the electronic properties of transition metal oxides is related to the appearance of superconductivity in some oxides of copper and bismuth [21].

$\mathrm{NiO}$ and $\mathrm{CoO}$ powders (Johnson Matthey) were pressed into pellets at room temperature and fixed with silver paint in scanning electron microscope (SEM) specimen holders. The samples were investigated by using the CL mode in a Hitachi S-2500 SEM at temperatures between 80 and $300 \mathrm{~K}$. Energy and current of the electron beam were $25 \mathrm{keV}$ and $10 \mathrm{nA}$ respectively. For panchromatic CL measurements the light arising from the sample was concentrated by an optical lens on a Hamamatsu H957-08 photomultiplier directly attached to a window of the microscope. To record spectra an optical guide was used to feed the light to an Oriel 78215 

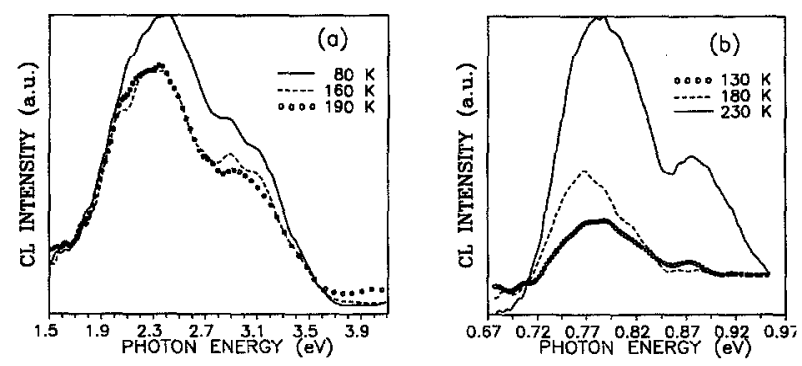

Fig. 1. Visible (a) and near infrared (b) CL spectra of $\mathrm{NiO}$ at several temperatures.

computer controlled monochromator with the photomultiplier placed at the exit split. Spectra in the infrared range $(800-1800 \mathrm{~nm})$ were recorded with a North Coast germanium detector.

Figure 1 shows visible and infrared CL spectra of $\mathrm{NiO}$ at different temperatures. Peaks are observed at $0.78 \mathrm{eV}, 0.87 \mathrm{eV}, 2.4 \mathrm{eV}, 2.9 \mathrm{eV}$ and $3.1 \mathrm{eV}$. In Fig. 2 the panchromatic C.I intensity in the visible range as a function of temperature is represented. CL intensity decreases by increasing temperature from $80 \mathrm{~K}$ up to about $190 \mathrm{~K}$. Then, CL intensity raises with further heating and after reaching a maximum at about $250-260 \mathrm{~K}$, decreases again with a new temperature increase. The appearance of this maximum in the mentioned temperature range has been found to be reproducible in different runs and different samples. Quantitative features, as the intensity of the peak relative to the intensity at $80 \mathrm{~K}$, are not reproducible. Visible and infrared spectra of $\mathrm{CoO}$ are shown in Fig. 3. The main peaks appear at $0.76 \mathrm{eV}$, $0.87 \mathrm{eV}, 2.65 \mathrm{eV}$ and $2.85 \mathrm{eV}$. The evolution of the panchromatic $\mathrm{CL}$ intensity in the visible range as a function of temperature is shown in Fig. 4. In the range

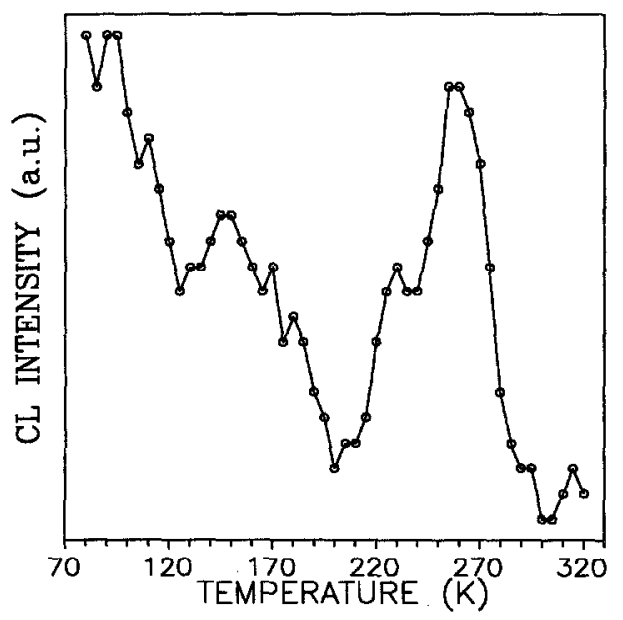

Fig. 2. Temperature dependence of NiO CL intensity in the visible range.
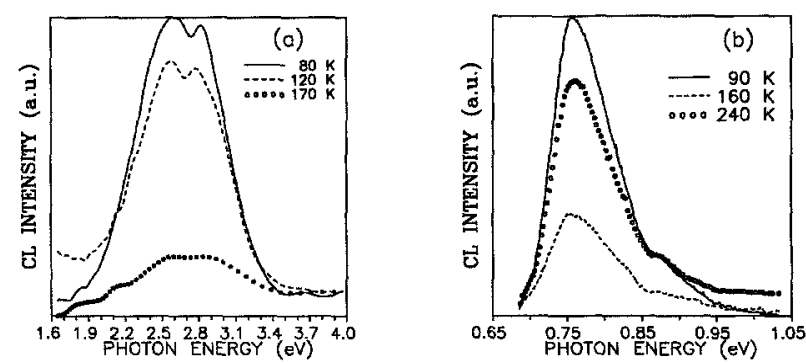

Fig. 3. CL spectra in the visible (a) and near infrared (b) ranges recorded from $\mathrm{CoO}$ at different temperatures.

$120-160 \mathrm{~K}$ a qualitatively reproducible peak is observed.

In our $\mathrm{CL}$ spectra of $\mathrm{NiO}$ the emission at about $4 \mathrm{eV}$ related to the charge transfer gap is not observed, probably due to the low efficiency of our detection system in the high energy range. The broad band peaked at about $2.4 \mathrm{eV}$, observed in the visible range spectra of Fig. 1(a), corresponds to the $2.5 \mathrm{eV}$ band reported by Tsu and Esaki [11]. The difference in peak energies observed in [11] and in this work can be explained by the lower temperature $(6 \mathrm{~K})$ of the measurements in the mentioned study. The spectra of Fig. 1(a) show a slight shift of the main peak to higher energies at low temperatures. At $80 \mathrm{~K}$ the maximum appears at about $2.47 \mathrm{eV}$. This peak was tentatively attributed by Tsu and Esaki to the magnetic exciton state $3 d^{8} 2 p$. In [12], the CL spectra of $\mathrm{NiO}$ films depend on the deposition conditions, showing a dominant band gap emission but only in some cases a band peaked near $2.4 \mathrm{eV}$. In Fig. 1(a) other bands appear as shoulders in the high energy side of the main $2.4 \mathrm{eV}$ band. The existence of a number of transitions at energies below the band gap in $\mathrm{NiO}$ have been often detected by different

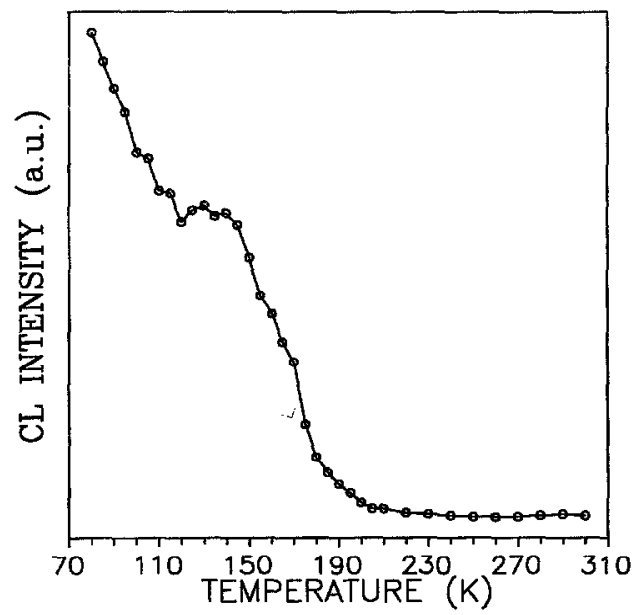

Fig. 4. CoO visible CL intensity evolution with temperature. 
techniques, mainly by optical absorption. Tsuboi and Kleeman [8] observed nine absorption bands, in the energy range $0.9-3.1 \mathrm{eV}$, associated to the $\mathrm{Ni}^{2+}$ ion. Adler and Feinleib [22] interpret a series of absorption bands below $4 \mathrm{eV}$ as pure $3 d^{8}$ intraionic transitions. The existence of intraionic transitions of the $3 d^{8}$ electrons of the $\mathrm{Ni}^{2+}$ ion at and below gap energies has been also observed in electron energy loss spectra [23]. Spin polarized electron energy loss spectroscopy $[24,25]$ revealed excitations of the $\mathrm{Ni}^{2+}$ ion in the range $0.6-2.8 \mathrm{eV}$ including transitions related to surface states. These and other previous results suggest that the $\mathrm{CL}$ emission observed here is due to transitions involving $3 d^{8}$ electrons of the $\mathrm{Ni}^{2+}$ ions.

$\mathrm{CL}$ spectra of $\mathrm{CoO}$ have been, to our knowledge, not previously reported. We attribute the existence of detectable luminescence in our experiments to the high excitation conditions in the SEM. The same $\mathrm{CoO}$ samples did not show PL emission even at $10 \mathrm{~K}$ [26]. The high energy part of the observed CL spectra could be due, according to the gap value of $2.5 \pm 0.3 \mathrm{eV}$ given in [5], to band gap or near band gap transitions. However, luminescence related to intraionic excitations cannot be ruled out. In fact, intraionic $d-d$ transitions at energies ranging from $0.80 \mathrm{eV}$ to $3.2 \mathrm{eV}$ have been derived from $a b$-initio calculations [27] and experimentally reported [23] in $\mathrm{CoO}$.

$\mathrm{NiO}$ and $\mathrm{CoO}$ are antiferromagnetic compounds with Néel temperatures of $523 \mathrm{~K}$ and $291 \mathrm{~K}$ respectively. As Figs 2 and 4 show, CL intensity was only detectable at temperatures well below $T_{N}$. In the above mentioned works on $\mathrm{MnO}$ the band interpreted as a magnon assisted luminescence vanishes at about $T_{N}$. The possibility of a similar behavior of our samples and the $\mathrm{MnO}$ could, for this reason, not be investigated. We will discuss the behaviour of the luminescence at low temperatures, compared with $T_{N}$, by considering previous results on other $\mathrm{Mn}$ antiferromagnetic compounds. Holloway et al. [28] reported large changes in the luminescence of $\mathrm{MnF}_{2}$ and $\mathrm{KMnF}_{3}$ at temperatures of about $T_{N} / 2$. The changes referred to frequency, intensity and lifetime of the luminescence emission. Smaller temperature dependent changes were observed in the same materials and other alkali manganese trifluorides near $T_{N}[29,30]$. In [28] and [29] the observed lumincscence shifts are cxplained by local lattice distortion around the excited $\mathrm{Mn}^{2+}$ ion due to magnetic ordering. It is assumed that magnetic ordering of the lattice influences the configuration coordinate diagram because the mean distance between an excited manganese ion and its nearestneighbor will depend upon whether the unexcited ions are magnctically ordcred. In more detailed configuration coordinate treatments $[30,31]$ it is proposed that the fluorescence shifts are consequence of a local magnetoelastic effect, which alters the local lattice distortion about the excited ion. This distortion is a function of the local spin orientations. The temperature at which the luminescence changes occur depends on the exchange energy for the excited-ion electron configuration.

The present results show that the previously described effect of magnetic ordering on the luminescence of manganese salts appear also in the case of the antiferromagnetic oxides $\mathrm{NiO}$ and $\mathrm{CoO}$. In particular, all these materials show anomalies in the curve of luminescence intensity as a function of temperature at about $T_{N} / 2$. This behaviour suggests that magnetic ordering effects could be also detectable in the luminescence properties of other compounds with antiferromagnetic properties as some high critical temperature superconductors (HTSC). Luminescence of HTSC has been often investigated in the past years. Besides luminescence emission related to impurity phases or unreacted precursors, the existence of intrinsic luminescence related to electronic processes in the superconductors materials is a well-established effect. CL spectra of different HTSC show bands in the blue-green spectral range which have been related to processes in the oxygen sublattice, e.g. [32-35]. CL microscopy reported in these and previous works has shown that the emission is inhomogeneously distributed in the HTSC samples, indicating an inhomogeneity of the oxygen content distribution. In particular, regions with higher CL emission correspond to oxygen depleted regions in $\mathrm{YBa}_{2} \mathrm{Cu}_{3} \mathrm{O}_{7-x}$ and $\mathrm{Tl}_{2} \mathrm{Ba}_{2} \mathrm{CuO}_{6+x}$ samples $[32,34,36]$. In the case of $\mathrm{YBa}_{2} \mathrm{Cu}_{3} \mathrm{O}_{7-x}$ there is a clear correlation between oxygen content and antiferromagnetic behavior with a Néel temperature of about $400 \mathrm{~K}$ for $x=1$, which decreases by increasing oxygen content [37-39]. In a previous work [32], we have measured the temperature dependence of $\mathrm{CL}$ intensity of $\mathrm{YBa}_{2} \mathrm{Cu}_{3} \mathrm{O}_{7-x}$ samples with the same experimental setup used here. The curves in some series of samples showed a peak of the CL intensity at about $220 \mathrm{~K}$, which was reproducible in different runs and different regions of the samples. We point out that this peak, which appears at about $T_{N} / 2$, could have the same physical origin that the luminescence anomalies reported in this and in previous works on antiferromagnetic compounds. In $\mathrm{YBa}_{2} \mathrm{Cu}_{3} \mathrm{O}_{7-x}$ the CL signal mainly arises from the oxygen deficient antiferromagnetic - regions which are present in the samples with high average oxygen content.

In summary, $\mathrm{NiO}$ and $\mathrm{CoO}$ show luminescence bands in the energy range in which intra-ionic transitions have been detected by other techniques or theoretically calculated. The dependence of the $\mathrm{CL}$ intensity as a function of temperature below $T_{N}$ reveals the existence of anomalies at about $T_{N} / 2$ similar to those reported for 
other antiferromagnetic compounds. This effect, which has been also observed in $\mathrm{YBa}_{2} \mathrm{Cu}_{3} \mathrm{O}_{7-x}$ is explained by the existence of magnetic ordering.

Acknowledgement-This work was supported by DGICYT (Project PB 93-1256).

\section{REFERENCES}

1. Hüfner, S., Steiner, P., Sander, I., Reinert, F. and Schmitt, H., Z. Phys., B86, 1992, 207.

2. Reinert, F., Steiner, P., Hüfner, S., Schmitt, H., Fink, J., Knupfer, M., Sandl, P. and Bertel, E., $Z$. Phys., B97, 1995, 83.

3. Zaanen, J., Sawatzky, G.A. and Allen, J.W., Phys. Rev. Lett., 55, 1985, 418.

4. Zheng Haoping, Phys. Rev., B48, 1993, 14868.

5. van Elp, J., Wieland, J.L., Eskes, H., Kuiper, P., Sawatzky, G.A., de Groot, F.M.F. and Turner, T.S., Phys. Rev., B44, 1991, 6090.

6. Low, W., Phys. Rev., 109, 1958, 247.

7. Newman, R. and Chrenko, R.M., Phys. Rev., 114, $1959,1507$.

8. Tsuboi, T. and Kleeman, W., J. Phys: Cond. Matter., 6, 1994, 8625.

9. Powell, R.J. and Spicer, W.E., Phys. Rev., B2, 1970, 2182.

10. Pratt, G.W. and Coelho, R., Phys. Rev., 116, 1959, 281.

11. Tsu, R, and Esaki, L., 10th Int. Conf. Phys. Semicond., p. 282-285. US Atomic Energy Commission, 1970.

12. Pundur, P.A., Sloceniece, E., Bets, V. and Kleperis, J., Latv. PSR Zinat. Akad. Vestis Fiz. Teh. Zinat. Ser., 3, 1986, 25 (in Russian).

13. Mochizuki, S., Piriou, B. and Dexpert-Ghys, J., $J$. Phys.: Condens. Matter., 2, 1990, 5225.

14. Mochizuki, S., Piriou, B., Dexpert-Ghys, J., Takayama, N., Kido, G., Mogi, I. and Suemoto, T., J. Phys.: Condens. Matter., 4, 1992, 6501.

15. Piriou, B., Dexpert-Ghys, J. and Mochizuki, S., $J$. Phys.: Condens. Matter., 6, 1994, 7317.

16. Mochizuki, S., J. Phys.: Condens. Matter., 2, 1990, 7225.

17. Mochizuki, S. and Takayama, N., J. Phys.: Condens. Matter., 3, 1991, 2729.

18. Reinen, D., Ber. Bunsenges. Phys. Chem., 69, 1965, 82.
19. Terakura, K., Williams, A.R., Oguchi, T. and Kübler, J., Phys. Rev. Lett., 52, 1984, 1830.

20. Jørgensen, C.K., Lenglet, M. and Arsène, J., Chem. Phys. Lett., 136, 1987, 475.

21. Torrance, J.B., Lacorre, P., Asavaroengchai, C. and Metzger, R.M., Physica, C182, 1991, 351.

22. Adler, D. and Feinleib, J., Phys. Rev., B2, 1970, 3112.

23. Gorschlüter, A. and Merz, H., Phys. Rev., B49, 1994, 17293.

24. Fromme, B., Schmitt, M., Kisker, E., Gorschlüter, A. and Merz, H., Phys. Rev., B50, 1994, 1874.

25. Fromme, B., Möller, M., Anschütz, Th., Bethke, C. and Kisker, E., Phys. Rev. Lett., 77, 1996, 1548.

26. García, J.A. and Remón, A., Private communication.

27. Shouheng Shi and Staemmler, V., Phys. Rev., B52, 1995, 12345.

28. Holloway, W.W. Jr., Kestigian, M., Newman, R. and Prohofsky, E.W., Phys. Rev. Lett., 11, $1963,82$.

29. Holloway, W.W. Jr. and Kestigian, M., Phys. Rev. Lett., 13, 1964, 235.

30. Holloway, W.W. Jr., Prohofsky, E.W. and Kestigian, M., Phys. Rev., 139, 1965, A954.

31. Prohofsky, E.W., Phys. Rev. Lett., 14, 1965, 302.

32. Gómez, P., Piqueras, J., Sayagués, M.J. and González-Calbet, J.M., Solid State Commun., 96, $1995,45$.

33. Díaz-Guerra, C. and Piqueras, J., J. Appl. Phys., 80, 1996, 998.

34. Díaz-Guerra, C., Piqueras, J. and Opagiste, C., Physica, C259, 1996, 121.

35. Díaz-Guerra, C. and Piqueras, J., Physica, C275, 1997, 37.

36. Gómez, P., Jiménez, J., Martín, P., Piqueras, J. and Domínguez-Adame, F., J. Appl. Phys., 74, 1993, 6289.

37. Tranquada, J.M., Moudden, A.H., Goldman, A.I., Zoliker, P., Cox, D.E., Shirane, G., Sinha, S.K., Vaknin, D., Johnston, D.C., Alvarez, M.S., Jacobson, A., Lewandowski, J.T. and Newsam, J.M., Phys. Rev., B38, 1988, 2477.

38. Ohno, T., Alloul, H., Mendels, P., Collin, G. and Marucco, J.F., J. Magn. Mag. Mat., 90-91, 1990, 657.

39. Picard, C. and Gerdanian, P., Solid State Commun., 87, 1993, 775 . 